\title{
Individual strategy update and emergence of cooperation in social networks
}

\author{
Carlos P. Roca*, ${ }^{*}$ Angel Sánchez ${ }^{\S, t, \ddagger}$ and José A. Cuesta ${ }^{\S}$ \\ ${ }^{*}$ Chair of Sociology, in particular of Modelling and Simulation, ETH Zürich, Switzerland

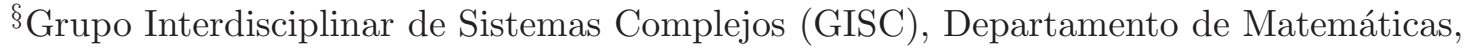 \\ Universidad Carlos III de Madrid, Spain \\ †Instituto de Ciencias Matemáticas CSIC-UAM-UC3M-UCM, Madrid, Spain \\ ‡Instituto de Biocomputación y Física de Sistemas Complejos (BIFI), \\ Universidad de Zaragoza, Spain
}

\begin{abstract}
In this paper, we critically study whether social networks can explain the emergence of cooperative behavior. We carry out an extensive simulation program in which we study the most representative social dilemmas. For the Prisoner's Dilemma, it turns out that the emergence of cooperation is very dependent on the micro-dynamics. On the other hand, network clustering mostly facilitates global cooperation in the Stag Hunt game, whereas degree heterogeneity promotes cooperation in Snowdrift dilemmas. Thus, social networks do not promote cooperation in general, because the macrooutcome is not robust under change of dynamics. Therefore, having specific applications of interest in mind is crucial to include the appropriate microdetails in a good model.
\end{abstract}

\section{Introduction}

More than thirty years ago, Schelling (1978) explored the relation between the behavior characteristics of the individuals comprising some social aggregate and the characteristics of that aggregate. Such an analysis, as opposed to the more traditional perspective of determining the effects of the collective properties on individual ones (Durkheim's (1964) social facts and forces), can be used in two different ways: predicting the aggregate behav-

This work has been supported by Ministerio de Ciencia e Innovación (Spain) through grant MOSAICO, and by Comunidad de Madrid (Spain) through grant MODELICO-CM. 
ior from the knowledge of the individual one (bottom-up) or, on the contrary, uncover the possible individual mechanisms compatible with an observed aggregate pattern (top-down) (Coleman, 1990). Both lines of research become complicated when the behavior of individuals influences and is influenced by other individuals and even by the whole collectivity.

A specific sociological context where the micro-macro link is particularly relevant is the study of social traps or dilemmas (Platt, 1973; Dawes, 1980). As Kollock (1998a) says (p. 183), "social dilemmas are situations in which individual rationality leads to collective irrationality. That is, individually reasonable behavior leads to a situation in which everyone is worse off than they might have been otherwise". This issue is actually at the core of long-standing problems such as the emergence of cooperation (Pennisi, 2005). Cooperation is behavior that is costly for the individual while ultimately benefiting other individuals or social aggregates. Paradigmatic models of cooperation within the framework of social dilemmas are the Public Goods game (also known as the Tragedy of the Commons (Hardin, 1968)) and the Prisoner's Dilemma (Axelrod, 1984). Both contain the game-theoretical ingredients of social dilemmas: a Nash equilibrium, a situation from which no player has incentives to deviate unilaterally, that is deficient, i.e., there are other sets of strategies in which everyone is better off (i.e., the Nash equilibrium is Rawls deficient (Rawls, 1971)).

One of the classical questions of social theory (Granovetter, 1985) is how behavior and institutions are affected by social relations. Recently, there is a huge literature on the role of social networks in the diffusion of behaviors and opinions in a society, and a variety of mechanisms through which this influence is exerted have been identified. Examples of these mechanisms include reciprocity and territoriality (Axelrod, 1984), reputation (Raub \& Weesie, 1990; Nowak \& Sigmund, 1998), the establishment of a group identity (Brewer \& Kramer, 1986; Kollock, 1998b), social learning (Macy, 1991), trust (Buskens \& Raub, 2002), projection (Kanazawa, 2007), group reciprocity (Karp, Jin, Yamagishi, \& Shinotsuka, 1993) and even group size (Olson, 1965; Dawes, 1980; Marwell \& Oliver, 1993). Other mechanisms, such as choice of partners (Yamagishi, Hayashi, \& Jin, 1994), costbenefit analysis (Jackson \& Wolinsky, 1996) or social plasticity (Eguiluz, Zimmermann, Cela-Conde, \& San Miguel, 2005), have led to the study of dynamic social networks, in which the individuals can change their links as a result of their interactions with their neighbors (see de Vos, Smaniotto, and Elsas (2001); Newman, Barabási, and Watts (2006) or Jackson (2008) for reviews). Here we will restrict ourselves to the case of static networks, that provide a good framework to study the problems arising with the micro-macro link, and constitute a key step to proceed later to the dynamic problem.

This paper focuses on the "strategy update rule" or ("update rule" for short): the manner in which individuals adapt their behavior as a function of that of their neighbors, i.e., the micro-dynamics. We will consider agents with limited rationality, who do not have memory, and whose communication with their neighbors is limited to observing their actions and the payoffs they receive. This implies that there are no reputation, reciprocity or identity effects. Admittedly, these are very simple rules, but their simplicity will allow us to gain insight on the micro-macro link in the context of explanations of cooperative 
behavior based on the existence of a social network. In fact, in the past few years, the general belief that networks generally support cooperation has grown among researchers of different fields (Cohen, Riolo, \& Axelrod, 2001; May, 2006). Here we critically examine this issue by considering a variety of update rules and of model networks. Our main research questions can be posed as follows:

Question 1: Do social networks support or otherwise enhance the emergence of cooperation among limited rationality agents?

Question 2: Does the observation of cooperation on models of social networks depend on the type of network, the strategy update rule of the agents, or the interplay of both?

We find it helpful to advance our main conclusion here: We will argue that the emergence of cooperation at the macro-level (social aggregate) due to an update rule (microdynamics) is largely dependent on the details of the network (i.e., on its micro-structure), and that different micro-structures may actually interact with the same update rule in different directions (i.e., promoting cooperation or inhibiting it). To support this conclusion, our paper is structured as follows: After a review of the most relevant precedents of this work, we show analytically that when there is no social substrate, all the strategy update rules we consider lead to the same collective behavior. We subsequently proceed to the situation when there is structure in the society, which we illustrate by presenting results from an extensive simulation program in which we study the most representative social dilemmas (Prisoner's Dilemma, Snowdrift, Stag Hunt (Kollock, 1998a)) under different micro-dynamics and on different model networks. We will see that the same rule may promote or inhibit cooperation depending on the type of network in which it acts; conversely, the same type of network may give rise to global cooperation or not depending on the strategy update rule governing the agents' behavior. Hence, the answer to question 1 will be that, generally speaking, it cannot be said that social networks support or promote cooperation. In particular cases, networks can be a mechanism promoting the emergence of cooperation but, and this answers question 2, such a promotion requires specifying the micro-level dynamics, as the resulting behavior depends on the interplay of both.

\section{Previous research}

Our approach to the research questions posed above originates from the pioneering work by Axelrod (1984). His description of the emergence of cooperation due to assortment of cooperating individuals and subsequent spreading over a non-cooperative population is worth quoting (p. 167):

Clusters of players were examined to see how the evolution of cooperation could have gotten started in the first place. Clusters allow a newcomer to have at least a small chance of meeting another newcomer, even though the newcomer themselves are a negligible part of the whole environment of the natives. Even 
if most of a newcomer's interactions are with uncooperative natives, a small cluster of newcomers who use reciprocity can invade a population of meanies.

In other words, if the invaders have even a small amount of social structure, they can prevail over non-cooperative individuals. While Axelrod then goes on to study more sophisticated social structures, including labels, reputation, regulation and territoriality, we will focus on social structure simply understood as a network of contacts that governs who interacts with whom. This is reminiscent of Giddens's social structure (Giddens, 1979) conceived as a pattern of interactions. It is also interesting to note that the emergence of cooperation in an iterated Prisoner's Dilemma through formation of clusters amounts to saying with Granovetter (1985) (p. 505):

Insofar as rational choice arguments are narrowly construed as referring to atomized individuals and economic goals, they are inconsistent with the embeddedness position presented here.

The idea of the network as a promoter of cooperation was later considered by Nowak and May (Nowak \& May, 1992). They considered agents without memory and with strategies "always cooperate" or "always defect" placed on a two dimensional spatial array. In each round they played the game only with their neighbors: the eight nearest ones (king's moves on a chessboard, or Moore neighborhood) and themselves. It is important to note that the agents used the same strategy with all their neighbors. After the interaction, individuals updated their strategy by imitating the most succesful one in their neighborhood if it yielded better payoff than their own (unconditional imitation, see below). Nowak and May simulated this system with a Prisoner's Dilemma with payoff matrix (payoffs for the row player are given, and $b>1$ ):

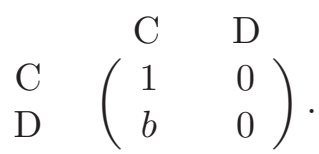

They found high cooperation even for large values of $b$, the temptation payoff received by a defector exploiting a cooperator. When cooperators prevailed, a fraction of the lattice sites were anyway occupied by defectors, who thrived in the perimeter of the cooperator clusters as described by Axelrod. Therefore, the mechanism worked even if agents had no memory, i.e., it appeared to be a direct consequence of the existence of a network of interactions and the corresponding possibility for cooperators to aggregate in clusters.

Another interesting work is due to Lomborg (1996). He simulated individuals (with short but nonzero memory) that played the Prisoner's Dilemma and evolved/learned by a proportional imitation rule (to be described below) applied to a subset of the population (which introduces implicitly some structure, albeit dynamical and not spatial). A vast majority of agents ended up belonging to one of two clusters: a large one of highly cooperative strategies (called nucleus by Lomborg) and a smaller one of more cautious strategies 
(referred to as shield). Such a split of the population was very long-lived not because of the individual success of its strategies when considered alone but because together they were able to prevail over the other.

Subsequently, Eshel, Samuelson, and Shaked (1998) analytically confirmed that cooperators can survive when interactions are local if they group together, so the benefits of cooperation are enjoyed primarily by other cooperators. They worked on simpler structures (Prisoner's Dilemma on one-dimensional rings) with unconditional imitation dynamics but discussed that their results also applied when mutations were introduced. Note that their choice for the update rule implies abandoning the rational agent paradigm of best response rules and substituting it by imitative agents. Interestingly, Eshel et al. showed that high costs of cooperation can lead to large cooperator density because for cooperation to succeed large clusters of cooperators are required.

Early in this century, Cohen et al. (2001) revisited this question. They studied lattices and random networks, both fixed and dynamical, in which individuals interacted with their four nearest neighbors only (von Neumann neighborhood). Their update rule was once again unconditional imitation. From their analysis, they concluded that what they called "context preservation" (the persistence of the interaction pattern) plays a more important role in the promotion of cooperation than "clustering" (which, in their case, refers to correlated interaction profiles instead of the widely accepted concept of transitivity).

Following these works, many other researchers undertook research along these lines. Far from establishing that a network of interactions promoted cooperation, a large number of papers have reported results which are to some extent contradictory. Interestingly, many of these works have been carried out with a physics perspective and appeared in physics journals, searching for a general pattern of behavior (recent reviews with many references on this issue are Szabó and Fáth (2007) and Roca, Cuesta, and Sánchez (2009b)). What we aim at showing here is that, for social dilemmas on spatial structures or, in general, on networks, there is no such a pattern, and that the observations of cooperative behavior depend intrinsically and fundamentally on the details of the model and, in particular, of the interplay of strategy update rule and network.

\section{Evolutionary games}

\section{Game-theoretical framework for social dilemmas}

We will consider symmetric $2 \times 2$ games, i.e., games among two individuals who choose between two strategies and with no difference in role. Using the same notation as above, the payoff matrix is:

$$
\begin{aligned}
& \text { C D } \\
& \mathrm{C} \quad\left(\begin{array}{cc}
1 & S \\
T & 0
\end{array}\right) \text {. }
\end{aligned}
$$

The strategies are labeled $\mathrm{C}$ and $\mathrm{D}$ for cooperate and defect, although the precise interpretation of what cooperation and defection mean depends on the specific social dilemma 
we consider. Indeed, certain values of $S$ and $T$ undermine mutual cooperation in different manners. To be specific, three scenarios are possible (Kollock, 1998a; Macy \& Flache, 2002):

- When $S<0$, a cooperator faces the risk of losing if the other player defects, performing worse than with mutual defection. In this situation, cooperation is dangerous and individuals are afraid of the consequences of being defected upon. When $T<1$, this is the Stag-Hunt game (see Skyrms (2003) for a thorough discussion), that has two equilibria: mutual cooperation, an optimal equilibrium (often called Pareto-dominant equilibrium), and mutual defection, which is deficient (often called risk-dominant equilibrium). The key issue in this dilemma is fear, i.e., whether the individuals can trust each other.

- When $T>1$, a cooperator is tempted to defect and obtain a payoff larger than that of mutual cooperation. The point is then that both individuals may then just do nothing (defect) hoping that the other will give in and cooperate, which may result in the worst possible outcome, mutual defection. Hence, the problem here is greed. When $S>0$ this is the Snowdrift or Chicken game (Sugden, 2004).

- Both tensions, fear and greed, are present when the two conditions are simultaneously satisfied, and then we are faced with the Prisoner's Dilemma (Axelrod, 1984). On the contrary, when none of the conditions is met, we have the Harmony game (Licht, 1999), which poses no dilemma in so far as mutual cooperation is the best outcome and is preferred by rational players.

\section{Model}

We will consider the above family of games in the context of a social network. In such a setting, every agent interacts with its neighbors in the network, i.e., the agents to whom it is linked. As in all previous works, the action taken by an agent is the same for all her neighbors. The neighborhood is also the agents' information set: they can see the actions and payoffs of their neighbors, and ignore everything about the rest of the agents in the network. Initially, agents will cooperate or defect with equal probability. Agents do not have memory and therefore actions and strategies coincide. At a simulation time step, all agents play the game simultaneously with all their neighbors and collect the corresponding accumulated payoff $\left(\pi_{i}\right)$. After the game stage, agents proceed to update their strategy, which they do by following a strategy update rule (to be described in the next subsection). A time step is then complete and the iteration is repeated. The network does not change during the simulation. This cycle is run as many times as it takes for the system to converge to a fixed strategy for all agents or to a stationary state in which the percentage of cooperators fluctuates around an approximately constant value. This percentage of cooperators is the magnitude we will monitor in our simulations.

\section{Strategy update/evolutionary rules}

A detailed review of all possible update rules and their specific applications would be beyond the scope of the present paper. For our present purposes we have decided to 
focus on three rules that have been widely used in previous research and in particular in the papers summarized above:

- Proportional imitation. This rule was first proposed by Helbing (1992) and Schlag (1998) and can be described as follows: Let $i=1 \ldots N$ label the individuals in the population. Let $s_{i}$ be the strategy of player $i, \pi_{i}$ her payoff and $N_{i}$ her neighborhood, with $k_{i}$ neighbors. One neighbor $j \in N_{i}$ is chosen at random and agent $i$ adopts the strategy of player $j$ with a probability that depends on the difference between the payoffs they obtained in the previous round through the expression

$$
p_{i j}^{t} \equiv \mathcal{P}\left\{s_{j}^{t} \rightarrow s_{i}^{t+1}\right\}= \begin{cases}\left(\pi_{j}^{t}-\pi_{i}^{t}\right) / \Phi_{i j}, & \pi_{j}^{t}>\pi_{i}^{t}, \\ 0, & \pi_{j}^{t} \leq \pi_{i}^{t},\end{cases}
$$

with $\Phi_{i j}=\max \left(k_{i}, k_{j}\right)[\max (1, T)-\min (0, S)]$ to ensure that $p_{i j}^{t} \in[0,1]$. In case the probabilistic decision turns out to be not to copy the neighbor's strategy, the player repeats her action in the next round. This is the rule used by Lomborg (1996).

- Unconditional imitation, which makes each player choose the strategy of the neighbor with the largest payoff, provided this payoff is greater than the player's. This is a deterministic rule, in contrast to the previous one, which is stochastic. This is the rule used by Nowak and May (1992), Eshel et al. (1998) and Cohen et al. (2001).

- Best response. This rule was introduced by Matsui (1992) and Blume (1993). Contrary to the previous two rules, that are imitative, i.e., in best response every player chooses her strategy as a best response to what her neighbors did in the last round with probability $p$ or leaves it unchanged with probability $1-p$ ( $p$ can be set to 1 to make the rule deterministic). Thus, best response schemes are a next step of sophistication in player capabilities as compared to simple imitation. In addition, best response is an innovative rule, as it allows extinct strategies to be reintroduced in the system whereas imitative dynamics cannot do that.

\section{Unstructured populations}

When there is no structure in the population, the classic framework to study the evolution of populations of cooperators and defectors is the replicator dynamics (Hofbauer \& Sigmund, 1998; Gintis, 2000), which assumes that every individual plays with every other (or, equivalently, that the social network of interactions is the complete graph). Let $x$ be the density of cooperators, and $f_{c}$ and $f_{d}$ the fitness of a cooperator and a defector, respectively. The replicator dynamics posits that $x$ evolves as (Hofbauer \& Sigmund, 1998) $(\dot{x}$ stands for $d x / d t)$

$$
\dot{x}=x(1-x)\left(f_{c}-f_{d}\right)
$$

Then, if cooperators are doing better than defectors their density rises accordingly, and the opposite occurs if they are doing worse.

For our social dilemmas, provided that the initial density of cooperators $x^{0}$ is different from 0 and 1 , the asymptotic outcome of the evolution is, for each game $\left(x^{*}\right.$ represents 


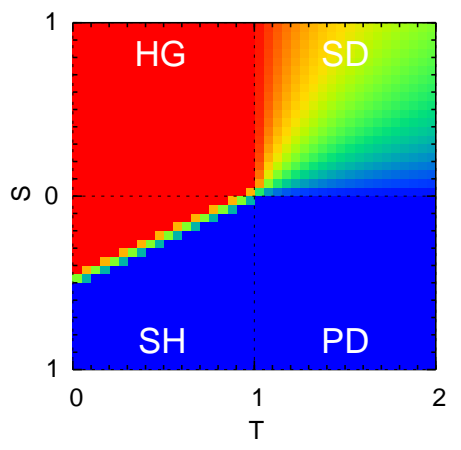

(a)

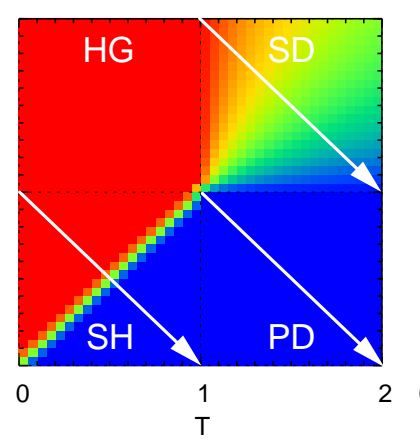

(b)

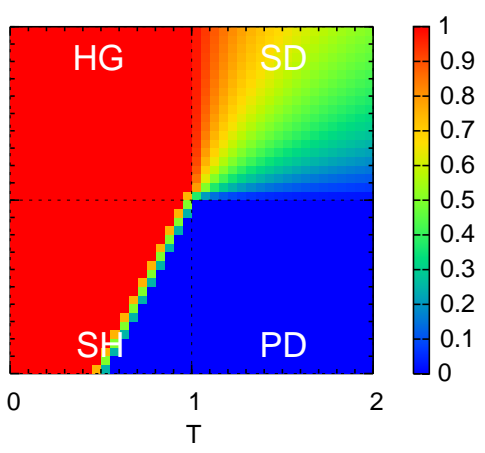

(c)

Figure 1: Standard results given by the replicator equation, Eq. (4), in infinite well mixed populations, for different initial conditions (cooperator densities $1 / 3,1 / 2,2 / 3$ ). The lines in plot (b) indicate the parameter regions that will be discussed in the remainder of the paper. The regions corresponding to each of the dilemmas are marked with initials: PD (Prisoner's Dilemma), SD (Snowdrift), SH (Stag Hunt), the remaining region corresponding to the Harmony Game (HG) where no dilemma appears.

the asymptotic density of cooperators) (Hofbauer \& Sigmund, 1998): Harmony, full cooperation, $x^{*}=1$; Prisoner's Dilemma, full defection, $x^{*}=0$; Stag Hunt, full cooperation if $x^{0}>x_{e}$, or full defection if $x^{0}<x_{e}$; Snowdrift, mixed population with $x^{*}=x_{e}$, regardless of the initial density $x^{0}$. For both Stag Hunt and Snowdrift games the coexistence equilibrium $x_{e}$, which is unstable for Stag Hunt but stable for Snowdrift games, has a value

$$
x_{e}=\frac{S}{S+T-1} .
$$

As a reference, Figure 1 shows the outcome of evolution under replicator dynamics for the four dilemmas presented above, depicted in the space of parameters $S$ and $T$. It also displays the lines that correspond to the game parameterizations that will be considered below.

The replicator equation is closely related to the proportional imitation rule, which we will use on structured populations. Proceeding as in (Lomborg, 1996; Gintis, 2000), it is straightforward to calculate the evolution with this update rule on an unstructured population. Let $n$ be the number of cooperators in a population of $N$ individuals, $0 \leq$ $n \leq N$. In one time step, the variation of $n$ will be given by the number of defectors that become cooperators minus the number of cooperators becoming defectors. Then, the expected value of the variation in the number of cooperators is ${ }^{1}$

$$
\mathcal{E}[\Delta n]=(N-n) \frac{n}{N} \frac{\left(f_{c}-f_{d}\right)_{+}}{\Phi}-n \frac{N-n}{N} \frac{\left(f_{d}-f_{c}\right)_{+}}{\Phi} .
$$

\footnotetext{
${ }^{1}(x)_{+}=x$ if $x \geq 0$, and $(x)_{+}=0$ if $x<0$.
} 
Defining $d x=\Delta n / N$ and $d t=\Phi^{-1}$, it results

$$
\mathcal{E}[\dot{x}]=x(1-x)\left(f_{c}-f_{d}\right)
$$

which is equivalent to Eq. (4) up to a time scale factor. Notice that the use of expected values means that, strictly speaking, this argument is only valid in the limit $N \rightarrow \infty$. For populations of finite size, the time evolution of $x$ will differ of Eq. (7) in a stochastic term, which in practice, however, is only relevant for very small population sizes (Traulsen, Claussen, \& Hauert, 2005), specially in what concerns the asymptotic state.

To study the case of an unstructured population driven by the unconditional imitation rule, it is enough to realize that, on a complete network, (i) all cooperators and all defectors obtain, respectively, the same payoff, and (ii) all players "scan" all others to identify the player with the maximum payoff and adopt her strategy. Hence, the population reaches a stationary state of full cooperation (full defection) if $f_{c}>f_{d}\left(f_{c}<f_{d}\right.$ ), in only one time step. Taking into account that the sign of $\dot{x}$ in Eq. (7) is determined by the term $\left(f_{c}-f_{d}\right)$, it is easy to conclude that this asymptotics is the same than the one induced by replicator dynamics in the Harmony game, the Prisoner's Dilemma and the Stag Hunt game. The behavior with the Snowdrift game presents an anomaly because the population "overreacts" when approaching the mixed equilibria at $x_{e}$, ending up in full cooperation (full defection) if $x^{0}<x_{e}\left(x^{0}>x_{e}\right)$. This pathology can be formally solved introducing a probability $p<1$ of changing strategy, which slows down this extremely fast dynamics, making the asymptotic fraction of cooperators fluctuate around $x_{e}$, with an amplitude bounded approximately by $\max \left(x_{e}, 1-x_{e}\right) p$.

Considering finally the third update rule, best response, a similar reasoning indicates that the best response for the current population state is simply the strategy that is achieving the highest payoff. Thus, as with unconditional imitation, the population reaches immediately a stationary state of full cooperation (full defection) if $f_{c}>f_{d}\left(f_{c}<f_{d}\right)$. As before, there are no differences with the asymptotics of replicator dynamics for the Harmony game, the Prisoner's Dilemma and the Stag Hunt game. With the Snowdrift game, the first time step drives the population to a state of full cooperation or full defection as in unconditional imitation, but instead of remaining fixed there, all the population keeps switching to the other strategy once per time step. As before, the formal solution is the introduction of a probability $p$ of change, which stabilizes the global fraction of cooperators around the mixed equilibrium, with the fluctuation bounded.

Thus, we have proven that the asymptotics reached on an unstructured population, i.e. on a complete network, with all the three strategy update rules is the same than with the replicator dynamics. Note, however, that proportional imitation is the only rule that also recovers the temporal evolution of replicator dynamics (recall, for instance, that for unconditional imitation we have shown above that convergence takes just one iteration). 

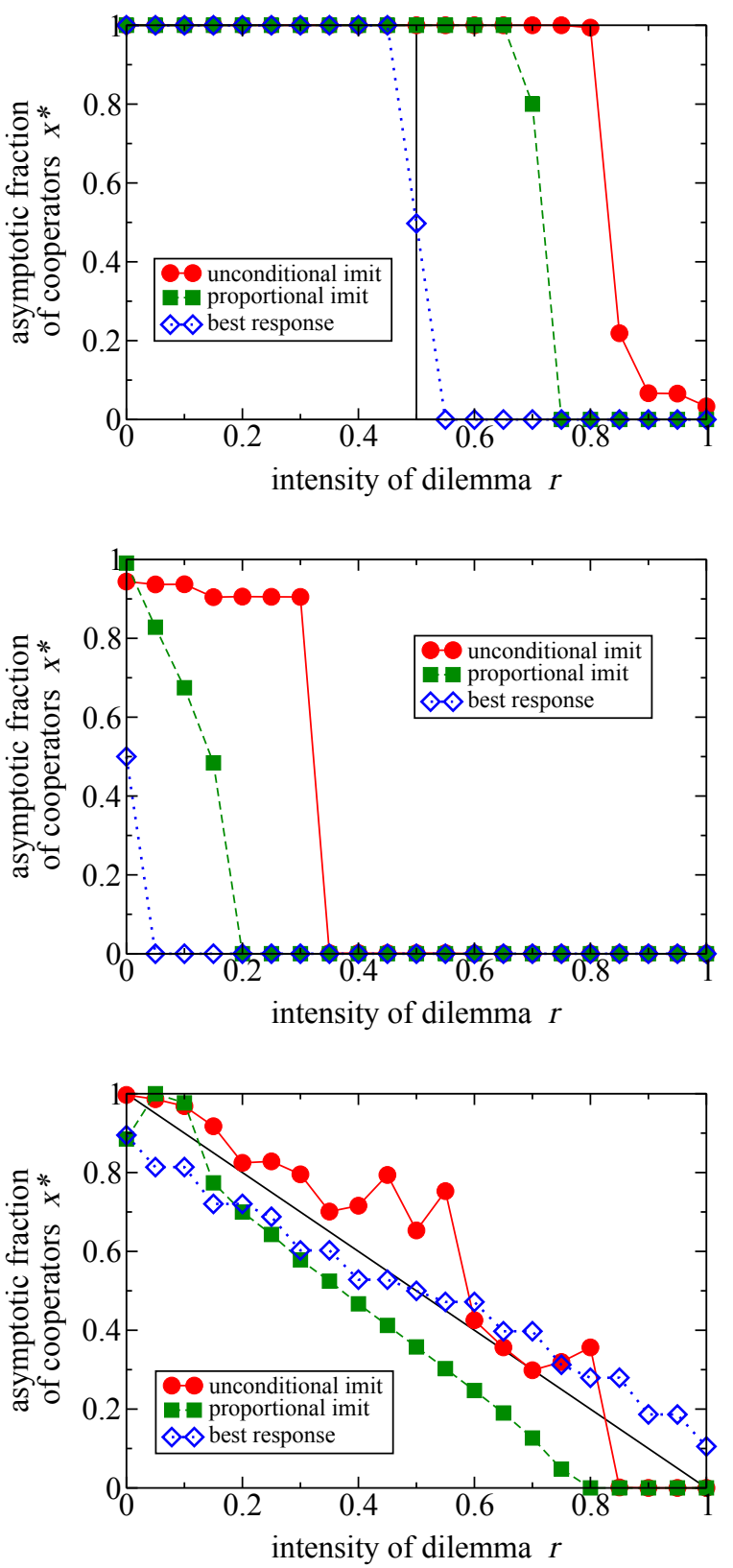

Figure 2: Cooperation asymptotics in a regular lattice of degree $k=8$ (Moore neighborhood), as a function of the intensity of dilemma $r$, for different social dilemmas. Top panel: Stag Hunt. Middle panel: Prisoner's Dilemma. Bottom panel: Snowdrift. Each panel shows results for three update rules: unconditional imitation (filled circles), proportional imitation (filled squares) and best response (empty diamonds). The results for well-mixed populations are shown as a solid line without symbols in Stag Hunt and Snowdrift games (for Prisoner's Dilemma the result is $x *=0$ for all the range of $r$ ). Other lines are a guide to the eye. For details on the simulations see Appendix. 


\section{Spatial networks}

We have run simulations (see Appendix for details of our numerical procedures) on square lattices of degree $k=8$, and our results are summarized in Fig. 2. Each panel of the figure presents results for a different region of the $S T$-plane corresponding to a different game. Specifically, for the Stag Hunt game we chose $(0 \leq r \leq 1)$

$$
T=r, S=-r
$$

for the Snowdrift game we chose

$$
T=1+r, S=1-r,
$$

and finally, for the Prisoner's Dilemma, we took

$$
T=1+r, S=-r
$$

These parameterizations of the game correspond to the three lines depicted in Fig. 1. The rationale for exploring along those lines is to increase the tensions in the different games by increasing the parameter $r$. Note that when $r=0$, in all three cases we are at the Harmony game, and proceed to stronger tensions as we take $r \rightarrow 1$.

As can be seen clearly from Fig. 2, the outcome of the simulations strongly depends on the strategy update rule, in all the three dilemmas. Thus, with unconditional imitation cooperation is enhanced in all cases (compare with the dashed line, which represents the well-mixed population, for reference; note that it overlaps with the $r$ axis for the Prisoner's Dilemma as the predicted cooperation is zero in that case), except for a minor region of large $r$ values in the Snowdrift game. With proportional imitation, cooperation is clearly enhanced in Stag Hunt, albeit to a lesser extent than in the unconditional imitation dynamics. In Snowdrift, cooperation is inhibited (except for low $r$ ) in Snowdrift, and it is promoted in Prisoner's Dilemma also only for low $r$. Finally, best response dynamics leads to results which are in general very similar to the well-mixed population, with both small promotion and inhibition of cooperation in the Stag Hunt and Snowdrift games, and a slight promotion for low $r$ in the Prisoner's Dilemma. Hence, we see that the effects of the micro-dynamics are most relevant is the Stag Hunt game, and that the promotion of cooperation in the Prisoner's Dilemma depends on the micro-dynamics, being maximum under unconditional imitation.

The reason for the sensitivity of the Stag Hunt to the dynamics is the existence of two competing equilibria in that case. In the well mixed population, convergence to a specific equilibrium depends on the amount of each of the strategies present in the initial population, and subsequently the convergence is global, i.e., all agents end up playing the same strategy. On the contrary, in the presence of spatial structure clusters converge locally to any of the two equilibria. Those regions where many cooperators are together 
yield them higher payoffs (they coordinate in the efficient equilibrium) and bring over the neighboring agents as time progresses.

The mechanism that explains the emergence of cooperation is the aggregation of cooperators facilitated by the spatial structure of the population, which leads to the formation and growth of clusters of cooperators. However, the key point we want to hightlight in this work is that this aggregation is very dependent on the update rule. With unconditional imitation clusters grow deterministically one network link each time step, and as consequence it is easy to see analytically that planar interfaces determine the conditions for cluster growth almost independently of the initial conditions. On the contrary, proportional imitation dynamics induces a much slower cluster growth with rough interfaces, that requires less tension (lower $r$ ) to develop. In this case, the result turns out to depend on the initial density of cooperators, in contrast to what occurs with unconditional imitation. Finally, with the best response rule, clusters are not stable from the start, because this rule is innovative and can reintroduce strategies that are not present within a cluster or a neighborhood at a given time.

Another important insight on the behavior of social dilemmas is the influence of clustering on the emergence of cooperation, in particular for proportional imitation. Indeed, the effect of this kind of regular lattices, along with imitative update rules, is directly linked to the first order correlation in the network, i.e. with the existence of triangles or clustering (defined as the ratio of the number of triangles present in the network over the number of all possible triangles that could be formed). The fact that neighbors of an individual are neighbors themselves is then crucial. To appreciate the effects of clustering, we present results for a random network of degree $k=8$ on the top panel of Fig. 3, which must be compared with the top panel of Fig. 2. Whereas the results for unconditional imitation and best response do not change much, for the random network the behavior under proportional imitation is basically the same as for the well mixed population. On the contrary, when the degree of both the square lattice and the random network is $k=4$ (implying zero clustering for both, i.e., no common neighbors), the results are comparable for all the dynamics [middle and bottom panels of Fig. 3].

The preceding comments do not contradict Cohen et al.'s discussion (Cohen et al., 2001) of clustering because they characterized clustering in terms of the average number of neighbors within a certain distance in order to highlight the existence of correlations within the network. They did not look at clustering understood as transitivity or existence of common neighbors: As a matter of fact, all their networks are $k=4$ lattices or are built in a random manner with the same average degree, and therefore they have zero clustering when measured by the density of triangles. In general, the effect of a reduced average distance between nodes is a shorter time of convergence to a very similar stationary state, and hence the asymptotic outcome for every social dilemma does not change (the interested reader is referred to (Roca, Cuesta, \& Sánchez, 2009a) for a discussion in depth of these issues). 

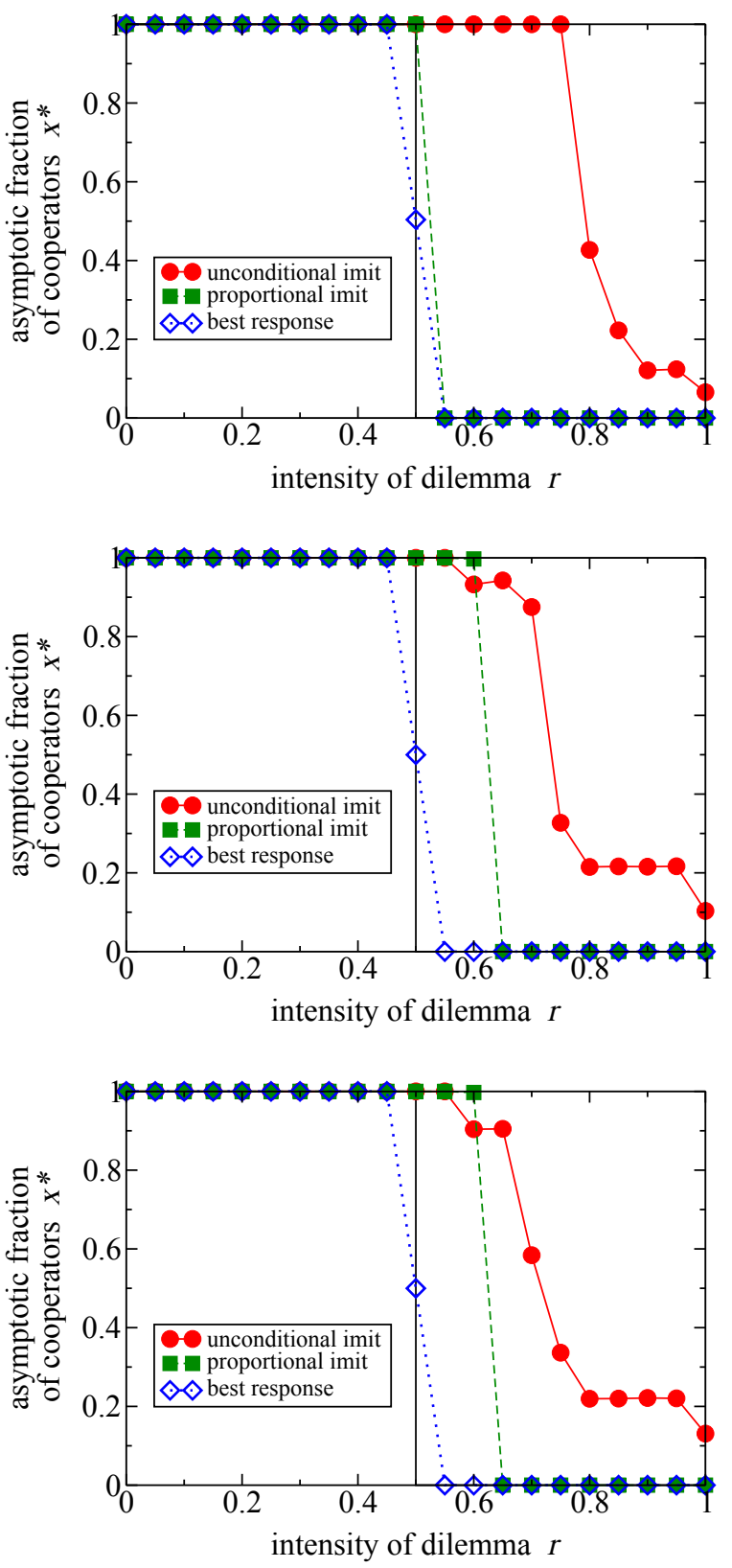

Figure 3: Cooperation asymptotics in Stag Hunt games on random networks and square lattices. Top panel: Random network, $k=8$. Middle panel: Square lattice, $k=4$. Bottom panel: Random network, $k=4$. Each panel shows results for three update rules: unconditional imitation (filled circles), proportional imitation (filled squares) and best response (empty diamonds). The results for well-mixed populations are shown as a solid line without symbols. Other lines are a guide to the eye. 
The work summarized in the previous section was focused on spatially structured populations (lattices) and random networks. The common feature to all these networks is that they are degree-homogeneous, meaning that they are regular (i.e., all nodes have exactly the same number of neighbors) or almost regular. In this section, we address the opposite case of highly heterogeneous networks, taking scale free networks (Albert \& Barabási, 2002) as our working example. Recent work has pointed out that these networks can be very favorable to cooperation (Santos, Pacheco, \& Lenaerts, 2006; Poncela, GómezGardeñes, Floría, \& Moreno, 2007) and can even self organize if they grow by incorporating new individuals who choose their neighbors depending on the payoffs of a game (Poncela, Gómez-Gardeñes, Floría, Sánchez, \& Moreno, 2008). Therefore, analyzing the micro-macro link on degree heterogeneous networks is an important subject that deserves attention both on its own and for its possible applications.

We carried out simulations for the three social dilemmas with the same parameters as in the preceding section. We generated scale free networks using a Barabási-Albert preferential attachment algorithm (Albert \& Barabási, 2002) with new nodes entering the network by connecting to $m=4$ preexisting individuals resulting on an average degree $k=8$. Our results are collected in Fig. 4. Contrary to our observations for spatial structure, in this case the game most severely affected is Snowdrift. On the other hand, the effect depends once again on the rule: Cooperation levels are dramatically increased with respect to the well mixed population, both for unconditional imitation and for proportional imitation; the latter leads to a smaller promotion than the former, but it is still quite large. Furthermore, there is also a noticeable effect on the Prisoner's Dilemma, which, albeit weak, is stronger than on Stag Hunt games. It is very interesting that in this case proportional imitation is the rule that leads to higher cooperation. Finally, as in degree-homogeneous networks, best response dynamics is the one that induces less differences with respect to the well mixed population.

We thus see very clearly that the effect of this kind of scale-free networks is qualitatively very different from that of spatial lattices, but in turn very dependent on the update rule. The reason for such a difference is the heterogeneity of degree, as we have checked that qualitatively similar results are obtained for other degree-heterogeneous networks. The basic microscopic mechanism that underlies this effect is a bias induced in the transitions from cooperator to defector, and viceversa, which are typical of Snowdrift games. In homogeneous networks these transitions happen with equal probability. In heterogeneous networks, however, for more connected individuals the transition from defection to cooperation becomes more probable than the opposite transition. The final result is that more connected individuals usually end up as the leaders of stable cooperative hubs. An important detail in this mechanism is that $S$ must be positive, i.e. there should not be risk in cooperation, for this effect to take place, which explains why the influence of heterogeneous networks is mostly concentrated on Snowdrift games. A detailed analytical study of this 

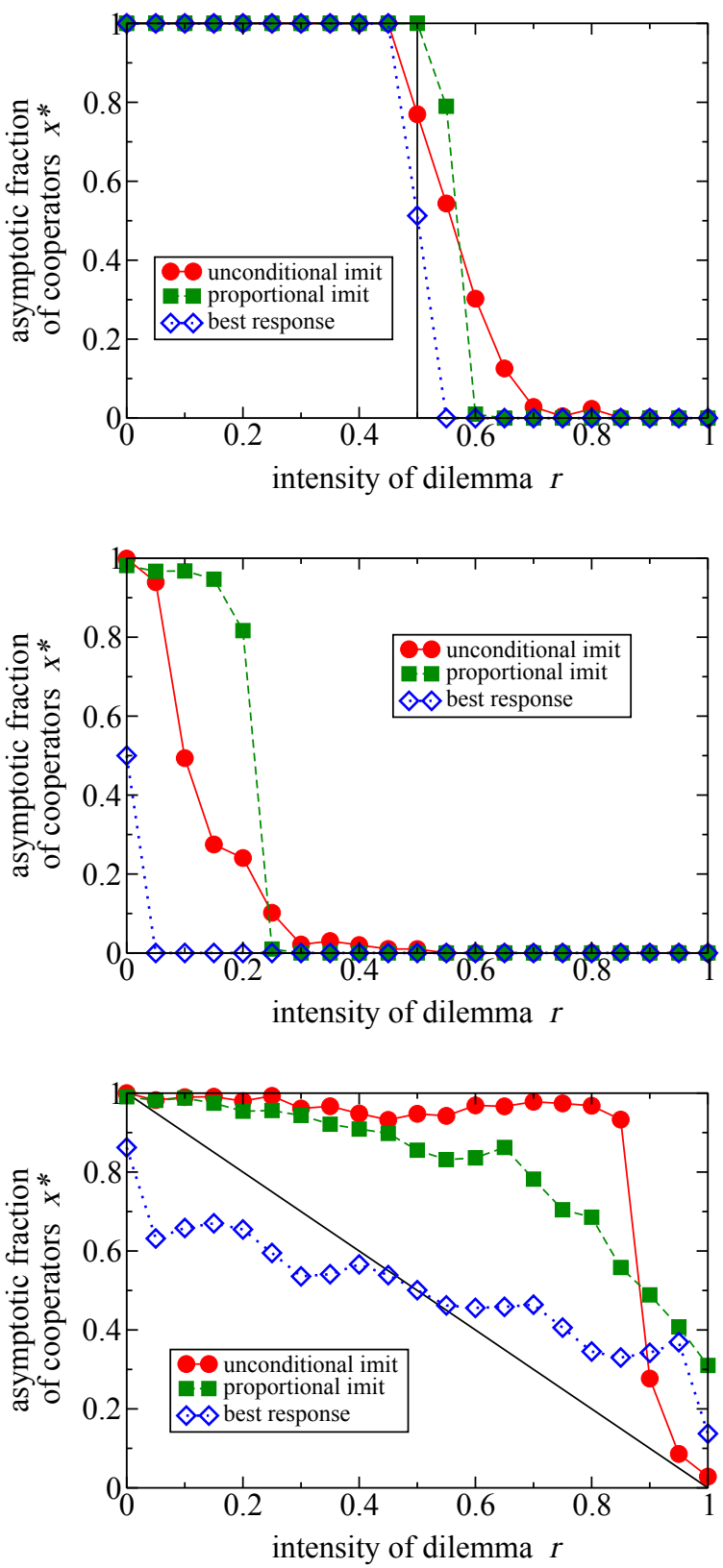

Figure 4: Cooperation asymptotics in a Barabási-Albert scale-free network of degree $k=8$, as a function of the intensity of dilemma $r$, for different social dilemmas. Top panel: Stag Hunt. Middle panel: Prisoner's Dilemma. Bottom panel: Snowdrift. Each panel shows results for three update rules: unconditional imitation (filled circles), proportional imitation (filled squares) and best response (empty diamonds). The results for well-mixed populations are shown as a solid line without symbols in Stag Hunt and Snowdrift games (for Prisoner's Dilemma the result is $x *=0$ for all the range of $r$ ). Other lines are a guide to the eye. 
issue for a toy model of cooperator and defector hubs separated by a set of individuals and with proportional imitation dynamics has been recently published (Floría, Gracia-Lazaro, Gardeñes, \& Moreno, 2009).

\section{Discussion and conclusions}

The most important conclusion of this work is that macro-level outcomes (emergence of cooperation, convergence to a given equilibrium) are very sensitive to changes in the evolutionary dynamics in the presence of a spatial or social structure in the population. This is in contrast with what we have seen in well mixed populations, i.e., in populations that lack social structure governing who interacts with whom, where the outcome is independent of the dynamics. However, as soon as the social structure is included in the model in terms of a network of interactions, each different micro-dynamics leads to a specific macro-outcome. This finding has very serious implications: In particular, it challenges the belief that social or spatial structures are in general supportive of global cooperative behavior, in so far as any promotion of cooperation is seen to depend on the micro-dynamics.

Regarding imitative dynamics, we have shown that they yield different results depending on their deterministic or stochastic nature. On degree homogenous networks, unconditional imitation, a deterministic rule, allows clusters of cooperators to grow until the cooperative behavior dominates the population even in a range of parameters of the Prisoner's Dilemma. On the contrary, with proportional imitation the promotion of cooperative behavior is clearly lower in the Prisoner's Dilemma, and even changed to inhibition in Snowdrift. The main influence of proportional imitation is then limited to the Stag Hunt game, due to the fact that in this dilemma there are two possible outcomes as discussed above.

On degree-heterogeneous (scale free) networks, the topology does not allow the formation of clusters, and another mechanism intervenes in the outcome of the microdynamics: the formation of cooperative groups around highly connected individuals or hubs. Under both imitative dynamics, these individuals end up being cooperators, and are henceforth able to bring their neighbors along to a cooperative state. Such a mechanism allows for a large promotion of cooperation in the Snowdrift game, reaching into a region of the Prisoner's Dilemma, with qualitative differences depending on the imitation rule considered. Cooperation in coordination games such as the Stag Hunt does not benefit much from this kind of networks under any of the imitative dynamics.

Therefore, clustering (understood as density of triangles or transitivity) and degree heterogeneity seem to be the two more important topological features of population structure with an impact on social dilemmas. Furthermore, these two characteristics lead to changes in the macro-outcome which are not the same under different update rules. This result has important consequences: If we only observe a given macro-state (say, global cooperation in a a Prisoner's Dilemma) we can not know whether models based on a degreeheterogeneous network under proportional imitation dynamics or on a degree-homogeneous 
network under unconditional imitation are the correct ones to describe our observations. Further research would then be needed to ascertain what is the individual behavior and the social structure underlying the aggregate of individuals.

Under best response dynamics, the social structure does not seem to play much of a role: Populations of all types tend to converge to the Nash equilibrium of the individual game. As this does not agree with observations of real systems and with many experiments, then we are faced with a puzzle: Either best response, with its alleged cognitive improvement with respect to imitation, is not well suited to model individual interactions, or the models need some rethinking. The absence of network effects under best response implies that the stability and efficiency of the two mechanisms we have discussed for the promotion of cooperation, namely cluster formation and cooperative hubs, requires imitation. This is so because innovative rules such as best response lead to strategy changes in the inner core of the cluster or on the hubs that eventually spread defection to the surroundings.

From the above discussion, a testable hypothesis emerges: a particular dynamics at the micro-level implies a non-trivial dependence of the macro-outcome on the kind of game and the concrete topology of the social network. Imitative update rules are only compatible, and hence verifiable in experiments, with the following behavior: games on lattices only display a clear influence of the network in the case of Stag Hunt games, whereas games on degree-heterogeneous networks do so only in Snowdrift games. In case experiments would provide positive evidence in this direction, then it would be possible to test the temporal evolution of the population, verifying the formation of clusters of cooperators with Stag Hunt games and the existence of cooperative hubs for Snowdrift games. A similar testable hypothesis can be posed for best response rules: best response as a micro-level dynamics implies a lack of influence of social structure for most games, they being Prisoner's Dilemmas, Stag Hunt or Snowdrift games.

Very recently, some experiments have dealt with the behavior of people playing the Prisoner's Dilemma on small (Kirchkamp \& Nagel, 2007; Traulsen, Semmann, Sommerfeld, Krambeck, \& Milinski, 2010) and large (Grujić, Fosco, Araújo, Cuesta, \& Sánchez, 2010) lattices, yielding new insights about the micro-dynamics which individuals actually follow. While there is not complete agreement between the interpretation of the three experiments, the fact that very low (but nonzero) levels of cooperation are observed in all of them makes very likely that unconditional imitation can be ruled out as a description of the way players update their strategies. Experiments designed to test other games are badly needed to make progress on this issue.

Regarding other choices for the update rules, we believe that they will all reflect the influence of different networks in idiosyncratic manners. For instance, work by Buskens and Snijders (2008) shows that simulations on small networks are not very sensible to their structure for a rule based on propensities to cooperate driven by instantaneous best responses, in line with our results here on larger networks. However, very different update rules, with a clear learning interpretation, lead to other outcomes. It is worth quoting work in progress by Galán, Izquierdo, Santos, and Sánchez (2010), who elaborate on previ- 
ous research on two-player games (Izquierdo, Izquierdo, Gotts, \& Polhill, 2007; Izquierdo, Izquierdo, \& Gotts, 2008) with reinforcement learning dynamics as used by Macy and Flache (2002): Using the Prisoner's Dilemma as an example, they observe that the asymptotic state of a two-player game with this dynamics is full cooperation for both players with transients around a mixed strategy equilibrium. On the contrary, the presence of a network of players renders the fully cooperative equilibrium unreachable for practical purposes. Interestingly, reinforcement learning leads to an outcome that is completely different from those we have reported for our three rules, further supporting our main conclusion. Research on whether other learning-type update rules, such as belief learning (Cheung \& Friedman, 1997) or experience-weighted attraction learning (Camerer \& Ho, 1999), lead to different outcomes from that of reinforcement learning would be a big step towards making a universal claim along the lines of our conclusion. In such a context, an interesting issue would be the possibility of distinguishing between reinforcement and belief learning on a social network, in the direction found for $2 \times 2$ games by van der Horst, van Assen, and Snijders (2010).

In this study, the information and interaction set are the same, i.e., agents interact with a given set of neighbors and have information about their actions and payoffs in the previous round. In general these two sets may differ. Recent work by Ohtsuki, Nowak, and Pacheco (2007) has shown that when two different networks are used, one for interaction (playing the game) and one for information (updating the strategy), the results change. It is worth noting that they report that the optimum setting for cooperation to emerge is when both graphs coincide. In the context of this paper, they use three different update rules (birth-death, death-birth and imitation) and once again find that the evolutionarily asymptotic state depends on the rule even within this generalized picture, in line with our claims here. Therefore, we believe that the fact that in this work we have only looked at the case in which the information and interaction sets are the same does not suppose any limitation to our main conclusion.

Finally, when model networks are substituted in the simulations of social dilemmas by real social networks obtained from empirical data, results are different even if the networks have the same global statistical features (Lozano, Arenas, \& Sánchez, 2008b, 2008a), a surprising result that has been shown to arise from the existence of topological traps on the network, mesoscopic structures similar to bottlenecks that make it difficult for successful strategies to propagate. All this points in the direction that modelling social interactions on social networks is by no means a straightforward task: it requires clear and specific questions and a precise specification of the scope of applicability of the results (what type of social aggregates, what type of dilemma involved, etc.). A promising avenue for research on social dilemmas using evolutionary games may be to let all relevant features coevolve, including the update rules themselves (Moyano \& Sánchez, 2009). With such an approach one might be able to show that some of the micro-dynamics or the social structures which are in principle possible do not actually appear or are not relevant in an evolutionary context, thus narrowing down the range of choices for the modeller. This is of course a 
highly speculative conjecture, but we believe it is worth exploring it in order to shed light on the dependence of the macro-outcomes on the micro-level.

\section{References}

Albert, R., \& Barabási, A.-L. (2002). Statistical mechanics of complex networks. Reviews of Modern Physics, 74, 47-97.

Axelrod, R. (1984). The evolution of cooperation. New York: Basic Books.

Blume, L. E. (1993). The statistical mechanics of strategic interaction. Games and Economic Behavior, 5, 387-424.

Brewer, M. B., \& Kramer, R. (1986). Choice behavior of social dilemmas: effects of social identity, group size and decision framing. Journal of Personal and Social Psychology, 50, 543-549.

Buskens, V., \& Raub, W. (2002). Embedded trust: Control and learning. Advances in Group Processes, 19, 167-202.

Buskens, V., \& Snijders, C. (2008). Effects of network characteristics on reaching the payoffdominant equilibrium in coordination games: a simulation study. University of Utrecht, ISCORE paper, 232.

Camerer, C. F., \& Ho, T. (1999). Experience-weighted attraction learning in normal form games. Econometrica, 67, 827-874.

Cheung, Y.-W., \& Friedman, D. (1997). Individual learning in normal form games: Some laboratory results. Games and Economic Behavior, 19, 46-76.

Cohen, M. D., Riolo, R. L., \& Axelrod, R. (2001). The role of social structure in the maintenance of cooperative regimes. Rationality and Society, 13, 5-32.

Coleman, J. (1990). Foundations of social theory. Cambridge, Massachusetts: Harvard University Press.

Dawes, R. M. (1980). Social dilemmas. Annual Reviews of Psychology, 31, 169-193.

Durkheim, E. (1964). The rules of sociological methods. New York: Free Press.

Eguiluz, V. M., Zimmermann, M., Cela-Conde, M. G., \& San Miguel, M. (2005). Cooperation and the emergence of role differentiation in the dynamics of social networks. American Journal of Sociology, 110, 977-1008.

Eshel, I., Samuelson, L., \& Shaked, A. (1998). Altruists, egoists and hooligans in a local interaction model. American Economic Review, 88, 157-179.

Floría, L. M., Gracia-Lazaro, C., Gardeñes, J. G., \& Moreno, Y. (2009). Social network reciprocity as a phase transition in evolutionary cooperation. Physical Review E, 79, 026106.

Galán, J. M., Izquierdo, L. R., Santos, J. I., \& Sánchez, A. (2010). Social dilemmas under reinforcement learning dynamics on networks. in preparation.

Giddens, A. (1979). Central problems in social theory: Action, structure and contradiction in social analysis. Berkeley: University of California Press.

Gintis, H. (2000). Game theory evolving. Princeton: Princeton University Press.

Granovetter, M. (1985). Economic action and social structure: The problem of embeddedness. American Journal of Sociology, 91, 481-510.

Grujić, J., Fosco, C., Araújo, L., Cuesta, J. A., \& Sánchez, A. (2010). Humans exhibit diverse behavior when playing a prisoner's dilemma in a large network. in preparation.

Hardin, G. (1968). The tragedy of the commons. Science, 162, 1243-1248.

Helbing, D. (1992). Interrelations between stochastic equations for systems with pair interactions. Physica A, 181, 29-52. 
Hofbauer, J., \& Sigmund, K. (1998). Evolutionary games and population dynamics. Cambridge: Cambridge University Press.

Izquierdo, L. R., Izquierdo, S. S., \& Gotts, N. M. (2008). Reinforcement learning dynamics in social dilemmas. Journal of Artificial Societies and Social Simulation, 11, 1.

Izquierdo, L. R., Izquierdo, S. S., Gotts, N. M., \& Polhill, J. G. (2007). Transient and asymptotic dynamics of reinforcement learning in games. Games and Economic Behavior, 61, 259-276.

Jackson, M. O. (2008). Social and economic networks. Princeton: Princeton University Press.

Jackson, M. O., \& Wolinsky, A. (1996). A strategic model of economic and social networks. Journal of Economic Theory, 71, 44-74.

Kanazawa, Y. (2007). The promotion and evolution of cooperation through projection: Implications for social dilemmas and trust. Journal of Mathematical Sociology, 31, 187-204.

Karp, D., Jin, N., Yamagishi, T., \& Shinotsuka, H. (1993). Raising the minimum in the minimal group paradigm. Japan Journal of Experimental and Social Psychology, 32, 231-240.

Kirchkamp, O., \& Nagel, R. (2007). Naive learning and cooperation in network experiments. Games and Economic Behavior, 58, 269-292.

Kollock, P. (1998a). Social dilemmas: the anatomy of cooperation. Annual Reviews of Sociology, 24, 183-214.

Kollock, P. (1998b). Transforming social dilemmas: group identity and cooperation. In P. Danielson (Ed.), Modeling rational and moral agents (pp. 186-210). Oxford: Oxford Univ. Press.

Licht, A. N. (1999). Games commissions play: 2x2 games of international securities regulation. Yale Journal of International Law, 24, 61-125.

Lomborg, B. (1996). Nucleus and shield: The evolution of social structure in the iterated prisoner's dilemma. American Sociological Review, 61, 278-307.

Lozano, S., Arenas, A., \& Sánchez, A. (2008a). Community connectivity and heterogeneity: clues and insights on cooperation on social networks. Journal of Economic Interaction and Coordination, 3, 183-199.

Lozano, S., Arenas, A., \& Sánchez, A. (2008b). Mesoscopic structure conditions the emergence of cooperation on social networks. PLoS ONE, 3, e1892.

Macy, M. W. (1991). Chains of cooperation: threshold effects in collective action. American Sociological Review, 56, 730-747.

Macy, M. W., \& Flache, A. (2002). Learning dynamics in social dilemmas. Proceedings of the National Academy of Sciences of the USA, 99, 7229-7236.

Marwell, G., \& Oliver, P. (1993). The critical mass in collective action: A micro-social theory. Cambridge: Cambridge Univ. Press.

Matsui, A. (1992). Best response dynamics and socially stable strategies. Journal of Economic Theory, 57, 343-362.

May, R. M. (2006). Network structure and the biology of populations. Trends in Ecology and Evolution, 21, 394-399.

de Vos, H., Smaniotto, R., \& Elsas, D. A. (2001). Reciprocal altruism under conditions of partner selection. Rationality and Society, 13, 139-183.

Moyano, L. G., \& Sánchez, A. (2009). Evolving learning rules and emergence of cooperation in spatial prisoner's dilemma. Journal of Theoretical Biology, 259, 84-95.

Newman, M., Barabási, A. László, \& Watts, D. (Eds.). (2006). The structure and dynamics of networks. Princeton: Princeton Univ. Press.

Nowak, M. A., \& May, R. M. (1992). Evolutionary games and spatial chaos. Nature, 359, 826-829. 
Nowak, M. A., \& Sigmund, K. (1998). Evolution of indirect reciprocity by image scoring. Nature, $393,573-577$.

Ohtsuki, H., Nowak, M. A., \& Pacheco, J. M. (2007). Breaking the symmetry between interaction and replacement in evolutionary dynamics on graphs. Physical Review Letters, 98, 108106.

Olson, M. (1965). The logic of collective action. Harvard: Harvard University Press.

Pennisi, E. (2005). How did cooperative behavior evolve? Science, 309, 93.

Platt, J. (1973). Social traps. American Psychologist, 28, 641-651.

Poncela, J., Gómez-Gardeñes, J., Floría, L. M., \& Moreno, Y. (2007). Robustness of cooperation in the prisoner's dilemma in complex networks. New Journal of Physics, 9, 184.

Poncela, J., Gómez-Gardeñes, J., Floría, L. M., Sánchez, A., \& Moreno, Y. (2008, Jun). Complex cooperative networks from evolutionary preferential attachment. PLoS ONE, 3, e2449.

Raub, W., \& Weesie, J. (1990). Reputation and efficiency in social interactions: An example of network effects. American Journal of Sociology, 96, 626-654.

Rawls, J. (1971). A theory of justice. Cambridge: Harvard University Press.

Roca, C. P., Cuesta, J., \& Sánchez, A. (2009b). Evolutionary game theory: temporal and spatial effects beyond replicator dynamics. Physics of Life Reviews, 6, 208-249.

Roca, C. P., Cuesta, J. A., \& Sánchez, A. (2009a). Effect of spatial structure on the evolution of cooperation. Physical Review E, 80, 046106.

Santos, F. C., Pacheco, J. M., \& Lenaerts, T. (2006). Evolutionary dynamics of social dilemmas in structured heterogeneous populations. Proceedings of the National Academy of Sciences of the USA, 103, 3490-3494.

Schelling, T. C. (1978). Micromotives and macrobehavior. New York: W. W. Norton.

Schlag, K. H. (1998). Why imitate, and if so, how? A boundedly rational approach to multi-armed bandits. Journal of Economic Theory, 78, 130-156.

Skyrms, B. (2003). The stag hunt and evolution of social structure. Cambridge: Cambridge University Press.

Sugden, R. (2004). Economics of rights, co-operation and welfare (second ed.). Hampshire: Palgrave Macmillan.

Szabó, G., \& Fáth, G. (2007). Evolutionary games on graphs. Physics Reports, 446, 97-216.

Traulsen, A., Claussen, J. C., \& Hauert, C. (2005). Coevolutionary dynamics: From finite to infinite populations. Physical Review Letters, 95, 238701.

Traulsen, A., Semmann, D., Sommerfeld, R. D., Krambeck, H.-J., \& Milinski, M. (2010). Human strategy updating in evolutionary games. Proceedings of the National Academy of Sciences of the USA, 107, 2962-2966.

van der Horst, W., van Assen, M. A. L. M., \& Snijders, C. C. P. (2010). Analyzing behavior implied by ewa learning: An emphasis on distinguishing reinforcement from belief learning. Journal of Mathematical Psychology, in press.

Yamagishi, T., Hayashi, N., \& Jin, N. (1994). Prisoner's dilemma networks: selection strategy versus action strategy. In U. Schulz, W. Albers, \& U. Mueller (Eds.), Social dilemmas and cooperation (p. 233-250). New York: Springer-Verlag.

\section{Appendix \\ Appendix: Simulation details}

All the simulations were performed for a population size of $N=10^{4}$. The initial density of cooperators was $x^{0}=0.5$ and the update of strategies was done synchronously. 
With synchronous update, all the individuals in the population play the game once with all their neighbours, compare their payoff with them and decide the new strategy for the next time step. Then, they all update their strategy at once and their payoff is set to zero before the next step. Using asynchronous updates does not change qualitatively the results except for a minor region of parameters in the Snowdrift game near the boundary with the Prisoner's Dilemma (Roca et al., 2009a).

The time of convergence in the simulations was $T=10^{4}$ rounds of the game per individual. If the population did not reach full cooperation or defection, an average of the cooperator density during the last tenth of the time evolution was used to obtain the asymptotic cooperator density. We checked that this time of convergence is enough to reach a steady state. For each choice of game parameters, 100 realizations were performed to obtain a final average value for the asymptotic density of cooperators.

Each realization started from a newly generated population, with strategies randomly assigned and the network, when applicable, also randomly built. The homogeneous random networks were built directly, assigning links randomly in the population, while ensuring an equal number of links for every individual. All the regular lattices were built with periodic boundary conditions. 